\title{
An Enhanced Adaptive Data Processing Technique for Computing Synchrophasor Measurements under Power System Frequency Variation
}

\author{
Hamada Almasalma \\ Rodrigo J. Albuquerque \\ Grenoble Institute of Technology \\ G2Elab Laboratory \\ Grenoble, France \\ Hamada.Almasalma@ense3.grenoble-inp.fr \\ Rodrigo.Albuquerque@g2elab.grenoble-inp.fr
}

\author{
Raphaël Caire \\ Nouredine Hadjsaïd \\ Grenoble Institute of Technology \\ G2Elab Laboratory \\ Grenoble, France \\ Raphael.Caire@g2elab.grenoble-inp.fr \\ Nouredine.Hadjsaid@g2elab.grenoble-inp.fr
}

\begin{abstract}
This work proposes a frequency-adaptive data processing technique for reliable synchrophasor measurements estimation under power system frequency variation. The proposed technique consists of four steps in which frequency estimation, filtering process, fast linear interpolation, and Radix-2 decimation-in-time (DIT) fast Fourier transform (FFT) approach are applied in a synergistic manner to provide accurate synchrophasor estimates. The sampling rate remains unchanged and the frequency measurement is estimated from the classical weighted least-squares approach, filtering process is executed by a Finite Impulse Response (FIR) filter bank, composed by lowpass and bandpass filters, whose goal is to separate all input signal harmonic components. The digital filter bank's size is limited by the desired harmonic component imposed by the 3-dB cut-off frequency, related to the analog anti-aliasing lowpass filter, and an optimized design has been employed to obtain symmetric coefficients and odd length (type 1 FIR filter). Fast linear interpolation based on trigonometric identities, using adaptiveangles related to the estimated frequency, is applied at each filter output data whose outcomes are used by the half-cycle Radix-2 DIT FFT approach to provide raw data in which the corrected synchrophasor measurements are obtained after the filter phase and amplitude compensations. The proposed technique aims also to evaluate the impact of the frequency variation on each harmonic component present in the input signal. Test signals with $\pm 3 \mathbf{~ H z}$ offset-nominal corrupted by harmonics components are applied to evaluate the technique's performance and the results demonstrate its capability in dealing efficiently with signals corrupted by spurious frequencies, being envisaged for potential applications involving M-Class PMU model.
\end{abstract}

Index Terms-Fast linear interpolation, filtering process, frequency estimation, synchrophasor measurements.

\section{INTRODUCTION}

Deployment of Phasor Measurement Units (PMU) for the first time allowed the real possibility of acquisition of the

This work was carried out as part of the SOGRID project (www.sogrid.com), co-funded by the French agency for Environment and Energy Management (ADEME) and developed in collaboration between participating academic and industrial partners. The authors would like to thank the CAPES Foundation of Brazil for their valuable support. positive-sequence voltage and current phasor estimates, frequency estimation, and Rate of Change of Frequency (ROCOF) with temporal precision based on the same GPS-UTC time stamp. The measurements provide by these devices ensure an effective way to monitor the real-time operation status of a wide-area measurement system (WAMS) improving substantially their reliability, observability and security [1][3]. Accurate synchrophasor measurements is directly related to the algorithms employed during the estimation process and several techniques that deal with the impact of the frequency variation in the synchrophasor estimation from sampled data have been proposed [4]-[7].

The Annex C of IEEE Std. C37.118.1 [8] provides some reference techniques to develop algorithms, however some evidences suggest that the data processing techniques based on the Recursive Discrete Fourier Transform (RDFT) and FFT with a fixed nominal clock have often been employed. These techniques are severely affected by off-nominal input signals that will produce non-synchronous sampling condition whose impact is a spurious energy across the frequency spectrum related to the discontinuity between the harmonic angular frequencies of the continuous time signal and the center of frequency bins. This pernicious phenomenon is well known as leakage effect and three classical techniques - weighing windows, frequency tracking and resampling - may be applied to overcome the underlying problem whose goal is to give back for the Fourier transform its inherent robustness to estimate the parameters of interest [9].

Weighing windows are functions that are multiplied by the sampled signal in the discrete-time domain to produce less ripples in frequency domain caused by the abrupt truncation of the signal outside the required observation interval. Generally, cosine windows are used to reduce the effects of the lack of synchronism between the signal period and the sampling period, however large frequency drifts may significantly reduce the performance of these windows. The basic idea of frequency tracking is to use the estimated frequency to synchronize the 
sampling rate to the power signal period. Practical applications claim for Digital Phase-Locked Loop (DPLL) system [10], however this technique could face difficulties with clock resolution and noise rejection.

Resampling techniques aim to create an adjusted samples sequence (amplitude corrections) whose sampling frequency is matched to the power system frequency to ensure that there are no errors in the synchrophasor estimates. Finite Impulse Response (FIR) filter can perform this task where a combination between digital interpolation and decimation are performed [11]. Cubic spline approach seeks to fit piecewise third-order polynomials to adjacent pairs of a set points. This interpolation technique could provide good results [12], however it may suffer with abrupt signal variations caused by high order harmonic components. It is important to mention that, without precaution, the classical resampling techniques cited above may increase considerably the computational burden. In this paper, a frequency-adaptive data processing technique for reliable synchrophasor measurements estimation under power system frequency variation is proposed. Four steps are performed in a synergistic manner - frequency estimation, filtering process, fast linear interpolation, and half-cycle Radix-2 decimation-in-time (DIT) FFT approach - to provide accurate synchrophasor estimates. The proposed technique uses an unchanged sampling rate and the frequency measurement is estimated from the classical weighted least-squares approach [1] based on the rate of change of phase angle relative to the offset from nominal frequency. The filtering process is executed by a Finite Impulse Response (FIR) filter bank, composed by lowpass and bandpass filters, whose goal is to separate all input signal harmonic components. The digital filter bank's size is limited by the desired harmonic component imposed by the 3-dB cut-off frequency, related to the analog anti-aliasing lowpass filter, and an optimized design has been employed to obtain symmetric coefficients and odd lengths (Type 1 FIR filter).

Once the harmonic components are separated, fast linear interpolation based on trigonometric identities is performed on every component in which adaptive-angles related to the estimated frequency are employed. The proposed technique aims to evaluate the impact of the frequency variation on each component present on the input signal whose outcomes are used by the half-cycle Radix-2 DIT FFT approach to provide raw data in which the corrected synchrophasor measurements are obtained after the accomplishment of the filter phase and amplitude compensations. Test signals in the range of $\pm 3 \mathrm{~Hz}$ offset-nominal corrupted by 5 harmonics (second harmonic to sixth, $100 \mathrm{~Hz}-300 \mathrm{~Hz}$ ), are applied to evaluate the technique's performance therefore a span of 6 cycles of the nominal power frequency have been applied to ensure the accuracy of results. The obtained estimates demonstrate its capability in dealing efficiently with signals corrupted by spurious frequencies, being envisaged for potential applications involving M-Class PMU model.

The remainder of this paper is organized as follows. In Section II, a detailed description of the proposed adaptive-frequency data processing technique is described. Section III provides the simulation and result analysis. In Section IV are presented the concluding remarks.

\section{Description of the Proposed AdAptive TECHNIQUE}

In this section, a brief overview of the frequency estimation process is explained. It is also presented and discussed the FIR filter bank design, and the mathematical formulation of fast linear interpolation using trigonometric identities and half-cycle Radix-2 DIT FFT approach. The description in this section is given for $f_{o}=50-\mathrm{Hz}$ nominal frequency, but the procedures could also be applied to $60-\mathrm{Hz}$ signals. A fixed sampling clock corresponding to a frequency $N_{s}=$ 16 samples/cycle times the nominal frequency is employed resulting in a Nyquist frequency of 8 samples/cycle (i.e, a maximum band-limited signal of $400 \mathrm{~Hz}$ for a $50-\mathrm{Hz}$ system). For practical applications, an additive lack of accuracy caused by non-ideality of instrumentation transformers can be found, however this feature has not been considered in this paper.

\section{A. Frequency estimation}

For proper functionality, the proposed technique requires knowledge of the power system frequency. Based on the state of the art proposed by [1], a decoupled frequency estimation process is performed by weighted least-squares approach due to its good performance even when the sampled data are corrupted by Gaussian random noise. The sampling clock is locked to the nominal frequency and the actual frequency is estimated from the rate of change of phase angle relative to the offset from nominal where phase angles over a span of six cycles have been used. Standard deviation of 0.01 radian on angles estimates, caused by sample noise, is considered and the frequency measurements remain with a very low average error around $0.23 \%$. Thus, in the remaining paper it is presumed that the power frequency can be estimated with high degree of accuracy. It is important to comment that a lot of methods with regard to frequency estimation can be found in the literature [13]-[15].

\section{B. FIR filter bank design}

The FIR filter bank design is based on the Parks-McClellan optimization technique to provide a stable performance and a linear phase response. The type 1 FIR filters have been chosen to give symmetric coefficients, odd length, and frequency response that has even symmetry about both digital frequencies $\Omega=0$ and $\Omega=\pi$. This even symmetry allows the frequency response to take on any value at these two critical frequencies, thus the envisaged lowpass and bandpass filters used to separate the input signal components can be implemented using this FIR filter type. Global parameters as passband gain of $-1 \mathrm{~dB}\left(a_{p}\right)$ and a stopband gain of $-60 \mathrm{~dB}$ $\left(a_{s}\right)$ are used in the design of all filters. The errors within the passband and stopband regions are specified as $\Delta_{p}$ and $\Delta_{s}$, respectively, whose values specify the maximum allowable ripples inside these regions [16] 


$$
\begin{gathered}
\Delta_{p}=1-10^{0.05 \times a_{p}} \\
\Delta_{s}=10^{0.05 \times a_{s}} .
\end{gathered}
$$

The maximum filter bank's size is limited by the desired harmonic component imposed by the analog anti-aliasing lowpass filter, therefore the maximum allowed bank's size is 8 filters (1 lowpass for the fundamental frequency and 7 bandpass for the harmonic components). However, for a correct application, the 3-dB cut-off frequency needs to be below of the Nyquist frequency. Thus, the proposed technique uses the maximum number of bandpass filters equal to 5 (second harmonic (Bandpass 1) to sixth (Bandpass 5)), with a cutoff frequency of $350 \mathrm{~Hz}$. The center frequencies are allocated at $100 \mathrm{~Hz}$ to $300 \mathrm{~Hz}$, respectively, however the bandpass regions need to involve the variation of $\pm 3 \mathrm{~Hz}$ offset-nominal, thus an enlargement of the bandpass region associated with the order of the harmonic component need to be taken into account. This characteristic will impact on the quantity of coefficients for each FIR filter, thus the constraint used to limit the filters order is based on the group delay $(\tau)$. The indices of the coefficients are limited to the range $-M / 2 \leq n_{c} \leq+M / 2$, where $n_{c}$ is the number of coefficients or the length of the FIR filter and $M$ is its order. A constant group delay $\left(\tau=\left(n_{c}-1\right) / 2\right)$ [16], for any frequency, can be understood as the delay of samples that will give for the FIR filter a causal characteristic which will imply the existence of a linear phase shift response. It is of paramount importance to mention that the overall response delay of a FIR filter is matched with the filter order, because the $M$ initial samples need to be rejected due to the transient behavior of the convolution process between the input sampled signal and the filter's coefficients. This overall delay is taken into account in which it has been stipulated that the order of the filters can not be greater than the number of samples in the span of cycles of the frequency estimation process to ensure a concatenation between the steps. Fig. 1 illustrates the amplitude and phase responses for each FIR filter of the bank whose outcomes are used during the final calculation of the synchrophasor measurements and the Table I summarizes the individual parameters of the filters (order, length, group delay, and transition bands).

TABLE I

FILTER PARAMETERS

\begin{tabular}{cccccc}
\hline \multirow{2}{*}{ FIR Filter } & \multicolumn{4}{c}{ Parameters } \\
\cline { 2 - 6 } & Order & Length & Group delay & Transition bands (Hz) \\
\hline Lowpass & 64 & 65 & 32 & \multicolumn{2}{c}{$[58$ 80] } \\
Bandpass 1 & 64 & 65 & 32 & {$[68-90]$} & {$[110-132]$} \\
Bandpass 2 & 88 & 89 & 44 & {$[122-139]$} & {$[162-178]$} \\
Bandpass 3 & 88 & 89 & 44 & {$[169-186]$} & {$[215-231]$} \\
Bandpass 4 & 88 & 89 & 44 & {$[216-233]$} & {$[268-284]$} \\
Bandpass 5 & 94 & 95 & 47 & {$[264-279]$} & {$[323-339]$} \\
\hline
\end{tabular}

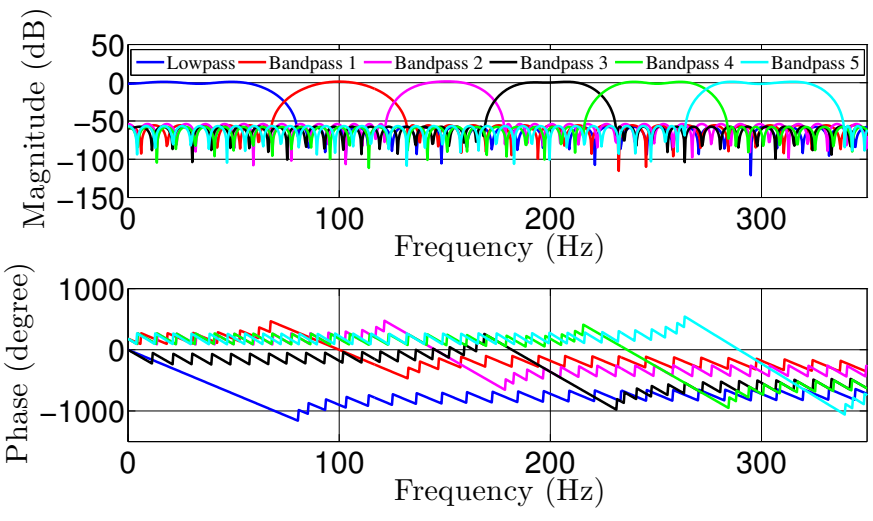

Fig. 1. Frequency response of the FIR Filter bank.

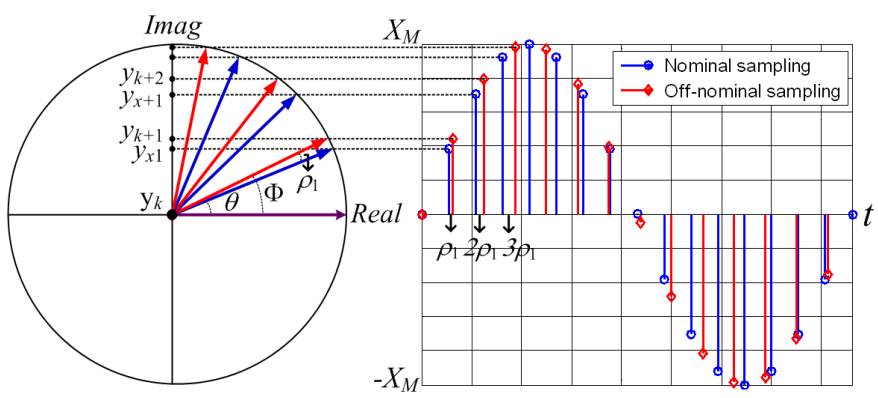

Fig. 2. Linear interpolation based on trigonometric identities.

\section{Fast linear interpolation}

Based on [1], a linear interpolation using trigonometric identities is applied on each filters output data. An extended version of this method have been implemented to take into account the effect of frequency variations on the sampled harmonic components. Off-nominal sampling condition will drift the output samples values with proportional variation to time, thus the goal is to modify the output sequence of the filter such that a new sequence synchronized with the estimated frequency can be obtained. The linear interpolation is a regression line that adjusts two points, given by (3)

$$
g(x)=\frac{b-x}{b-a} \times f(a)+\frac{x-a}{b-a} \times f(b)
$$

where $f(a)$ and $f(b)$ are the corresponding values for a generic function $f(x)$ at $x=a$ and $x=b$, respectively. The idea depicted in Fig. 2 is to calculate the adjusted output samples of the FIR lowpass filter taking projections of the sample amplitudes obtained by the off-nominal sampling condition over the trigonometric circle imaginary axis. Assuming that at instant $k$ and $k+1$ the off-nominal amplitudes $y_{k}$ and $y_{k+1}$ are available the adjusted amplitude $y_{x_{1}}$ can be determined using the following identities

$$
\begin{gathered}
\rho_{1}=\Phi-\theta \\
f(a)=y_{k}, f(b)=y_{k+1} \\
b-x=y_{k+1}-y_{x_{1}}=\sin \left(\rho_{1}\right) \times X_{M}
\end{gathered}
$$




$$
\begin{gathered}
b-a=y_{k+1}-y_{k}=\sin (\Phi) \times X_{M} \\
x-a=y_{x_{1}}-y_{k}=\sin (\theta) \times X_{M}
\end{gathered}
$$

thus,

$$
y_{x 1}=\frac{\sin \left(\rho_{1}\right)}{\sin (\Phi)} y_{k}+\frac{\sin (\theta)}{\sin (\Phi)} y_{k+1}
$$

being $\Phi=2 \pi\left(f_{o}+\Delta_{f_{o}}\right) / N_{s} f_{o}$ radians the off-nominal sampling interval expressed according to the estimated frequency; $\theta=2 \pi / N_{s}$ radians is the nominal sampling interval; and $\rho_{1}$ is the adaptive angle related to the estimated frequency given by the relative position difference between the nominal and off-nominal samples. The $\rho_{1}$ angle varies linearly to time, as illustrated in Fig. 2, therefore to compute the subsequent adjustable samples $\rho_{1}$ needs to be subtracted from the $\theta$ angle in (9). The first sample of the adjusted set will be numerically equal to the first filter output sample. Only two output samples of the filter are required to compute the adjusted sample being suitable for on-line implementation. An interesting feature is that all trigonometric identities are valid independent of the rotating phasor initial angle.

An extended version of this method have been implemented to enable the interpolation of the bandpass filter output samples. The basic idea is the same, however, special care must be taken to determine the variables of interest. The angular frequency of the harmonic component will require adaptive angles proportional to the harmonic order, thus

$$
\begin{aligned}
& \rho_{2}=2 \Phi-2 \theta ; \rho_{3}=3 \Phi-3 \theta ; \rho_{4}=4 \Phi-4 \theta ; \\
& \rho_{5}=5 \Phi-5 \theta ; \rho_{6}=6 \Phi-6 \theta
\end{aligned}
$$

furthermore, for performing a correct linear interpolation the nominal and off-nominal sampling intervals also need to be based on the harmonic order. Therefore, the generalized equation to compute the adjusted samples for the bandpass FIR filter is given by

$$
y_{x_{n}}=\frac{\sin \left(\rho_{n}\right)}{\sin (n \Phi)} y_{k_{n}}+\frac{\sin \left(n \theta-\rho_{n}\right)}{\sin (n \Phi)} y_{(k+1)_{n}}
$$

where $\rho_{n}$ is the adaptive angle according to $(10) ; n$ is an integer number representing the harmonic order (two to six); $n \Phi=2 \pi n\left(f_{o}+\Delta_{f}\right) / N_{s} f_{o}$ radians is the off-nominal sampling interval for the respective harmonic component expressed according to the estimated frequency; $n \theta=2 n \pi / N_{s}$ radians is the nominal sampling interval of the harmonic; $y_{k_{n}}$ and $y_{(k+1)_{n}}$ is the pair of samples taken at instant $k$ for each harmonic which goal is to compute the adjusted samples $y_{x_{n}}$.

\section{Half-cycle Radix-2 DIT FFT approach and frequency response compensation}

A half-cycle Radix-2 DIT FFT aproach have been employed for computing raw synchrophasor measurements using the adjusted output samples of the filters. DIT process is based on splitting the time domain sequence into even and odd samples in order to decompose discrete Fourier transform (DFT) into smaller DFTs whose calculation requires less computational effort [17], [18]. It uses the element called twiddle factor, $W_{N_{s}}^{n K}=e^{-j 2 n \pi k / N_{s}}$, to rewrite half-cycle DFT, given by (12)

$$
Y(K)=\sum_{n=0}^{\frac{N_{s}}{2}-1} y[n] W_{N_{s}}^{n K}
$$

By splitting $y[n]$ into even and odd samples, Equation (12) can be written as

$$
Y(K)=\sum_{n=0}^{\frac{N_{s}}{4}-1} y[2 n] W_{N_{s}}^{2 n K}+W_{N_{s}}^{K} \sum_{n=0}^{\frac{N_{s}}{4}-1} y[2 n+1] W_{N_{s}}^{2 n K} .
$$

Using the fact that $W_{N_{s} / 2}^{n K}=W_{N_{s}}^{2 n K}$, Equation (13) can be reformulated as (14). Where $A(K)$ is the DFT of evennumbered adjusted samples and $B(K) W_{N_{s}}^{K}$ is the DFT of odd-numbered samples

$$
\begin{aligned}
Y(K) & =\sum_{n=0}^{\frac{N_{s}}{4}-1} y[2 n] W_{N_{s} / 2}^{n K}+W_{N_{s}}^{K} \sum_{n=0}^{\frac{N_{s}}{4}-1} y[2 n+1] W_{N_{s} / 2}^{n K} \\
& =A(K)+B(K) W_{N_{s}}^{K} .
\end{aligned}
$$

Using the periodicity and symmetry properties, the following relations can be found: $A\left(K+N_{s} / 2\right)=A(K)$ and $B(K+$ $\left.N_{s} / 2\right)=B(K) ; W_{N_{s}}^{K+\left(N_{s} / 2\right)}=-W_{N_{s}}^{K}$, respectively. Thus, Equation (14) can be written for synchrophasor representation as

$$
Y_{s}(K)=\frac{2 \sqrt{2}}{N_{s}}\left(A(K)+B(K) e^{-j \frac{2 \pi K}{N_{s}}}\right)
$$

where $K$ varies from 1 to $N_{s} / 2$. The advantages of the half-cycle Radix-2 DIT FFT are remarkable because it uses a fractional data window, i.e. only half of the samples of the adjusted output set of each filter could be employed for fast computing of raw synchrophasor measurements, the parameters $e^{-j 2 \pi K / N_{s}}$ could be pre-calculated and stored for use in real time, and it is also possible to notice the correlation between the frequency parameter $K$ and the maximum allowed frequency of the band-limited input signal.

Using the frequency response of the FIR bank for each filter, in the form of a complex valued function of frequency, the amplitude and phase compensations can be performed for any particular frequency in the range of $\pm 3 \mathrm{~Hz}$ offset-nominal. The complex value for the frequency response is determined and converted to polar form, as

$$
H_{K}\left(e^{j \Omega}\right)=V_{K}(\Omega) \angle \Psi_{K}(\Omega) .
$$

In (16), $V_{K}(\Omega)$ and $\Psi_{K}(\Omega)$ represent the compensation in amplitude and phase, respectively, that the raw synchrophasor estimates will experience due to the flow of the samples through each filter. Therefore, the corrected synchrophasor estimates will be computed by

$$
Y_{s}(K)_{\text {corrected }}=\frac{Y_{s}(K)}{H_{K}\left(e^{j \Omega}\right)} .
$$




\section{Simulations AND Results}

This section presents the simulation and result analysis for a power system with $50 \mathrm{~Hz}$ nominal frequency. The proposed technique has been implemented using MATLAB 2014a environment. Test cases are performed in three parts: the starting point are off-nominal single-phase sinusoidal test signals (in pu) whose frequencies are located at critical boundaries of the $\pm 3 \mathrm{~Hz}$ range and these signals are corrupted with harmonic components until the sixth order. In the second, it is assumed an off-nominal input signal, also corrupted by spurious harmonic components, with a superimposed DC offset. In the third case, the performance between DFT approach and the proposed technique is compared for a fundamental test signal with frequency deviation. It is important to mention that the actual input signal frequency has been tracked by the frequency estimation process. Test signals for the case I are based on (18) being $k$ a given instant of time and $T_{\text {samp }}$ represents the sampling period.

$$
\begin{aligned}
& x(k)=x_{1} \cos \left(2 \pi\left(f_{o}+\Delta_{f}\right) k T_{\text {samp }}+\varphi(k)\right) \\
& +\sum_{m=2}^{6} x_{m} \cos \left(2 \pi m\left(f_{o}+\Delta_{f}\right) k T_{s a m p}+\varphi_{m}(k)\right)
\end{aligned}
$$

In the simulation, the harmonic component amplitudes are in the order of $10 \%, 7.5 \%, 9 \%, 11.5 \%$, and $6 \%$ related to the off-nominal signal amplitude, respectively. The $\varphi$ angle is equal to $-\pi / 4$ radians and the $\varphi_{m}$ angle is equal to $\pi / 8$, $\pi / 12, \pi / 3, \pi / 9$, and $\pi / 2$ radians according to the harmonic sequence. Total Vector Error (TVE) metric is the measure of error between the theoretical synchrophasor value of the signal being measured and the synchrophasor estimate, as (19). It must be less than $1 \%$ to comply with the IEEE Standard C37.118.1 [8]. Fig. 3 shows the TVE results given by the proposed technique for each component of the test signals which values satisfactorily fulfills the requirements of the standard.

$$
T V E(k)=\frac{\left|\vec{X}_{\text {estimate }(k)}-\vec{X}_{\text {theoretical }(k)}\right|}{\left|\vec{X}_{\text {theoretical }(k)}\right|} \times 100 \% \text {. }
$$

The signal used in the case II has an estimated frequency of $50.5 \mathrm{~Hz}$. A DC offset equivalent to $0.5 \%$ of the off-nominal signal amplitude is considered in (18), thus the assumed signal is $x_{D C}(k)=x_{D C}+x(k)$. The $\varphi$ angle is equal to $-\pi / 18$ radians, in addition spurious harmonic components with amplitudes equal to $12.5 \%, 8.5 \%, 11 \%, 7.5 \%$, and $7 \%$ and $\varphi_{m}$ angle equal to $\pi / 10,-\pi / 19,2 \pi / 9,3 \pi / 2$, and $-\pi / 22$ radians have been applied, respectively. Fig. 4 gives the TVE results for each signal component which values are also below the limit stated in the IEEE Standard. A slight increase in the TVE error of some components is reported, thus signals with very large DC offset and very small harmonic amplitudes, not particularly found in power systems, could reduce the performance of the proposed technique. For the case III, an off-nominal test signal without any harmonics and DC offset

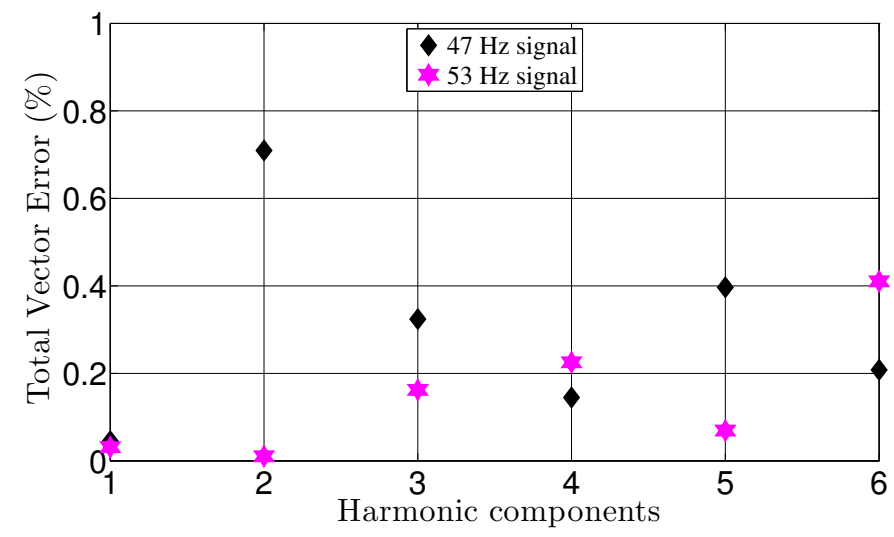

Fig. 3. Full span TVE error of the harmonic components for the frequency variation boundaries.

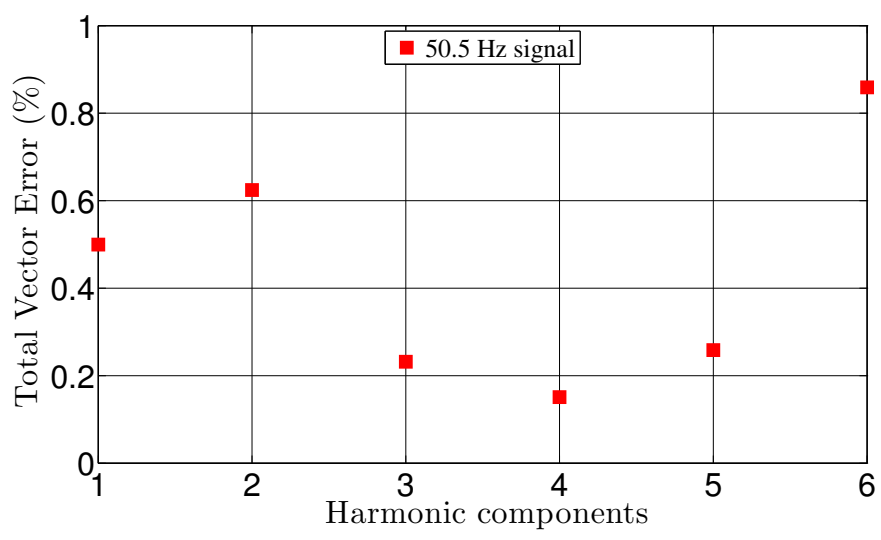

Fig. 4. Full span TVE error of the harmonic components for a $50.5 \mathrm{~Hz}$ signal with a DC offset equivalent to $0.5 \%$ of the off-nominal signal amplitude.

is used to compare the performance between the DFT approach and the proposed technique. Consider a reference sinusoid having a rms value of 1 at a frequency of $51 \mathrm{~Hz}$. The assumed phase angle of the synchophasor is $\pi / 4$ radians, so that the correct phasor representation of this signal is $\vec{X}=1 e^{j \pi / 4}$. Fig. 5 shows that the classical full cycle DFT approach gives results corrupted by a second harmonic component that will cause oscillations varying with time on the amplitude of synchrophasor estimate, which agrees with [1]. Filtering the DFT results through a post-processing averaging digital filter (ADF) the amplitude oscillations can be alleviated. A FIR filter with six equal coefficients has been employed and the convolution result is displayed. The estimate of a full span of the proposed technique is stable and it provides accurate magnitude estimates that are not affected by pernicious oscillations caused by frequency drift. This clearly shows that the interpolation process executed at the output FIR lowpass filter has properly adjusted the samples. In Fig. 6, a similar analysis is performed for the angle estimates which results arising from the full cycle DFT are also affected by the oscillations. Using the average FIR filter a small amount of residual second harmonic ripple can be obtained, however the angle of synchrophasor estimate contains an average slope corresponding to frequency 


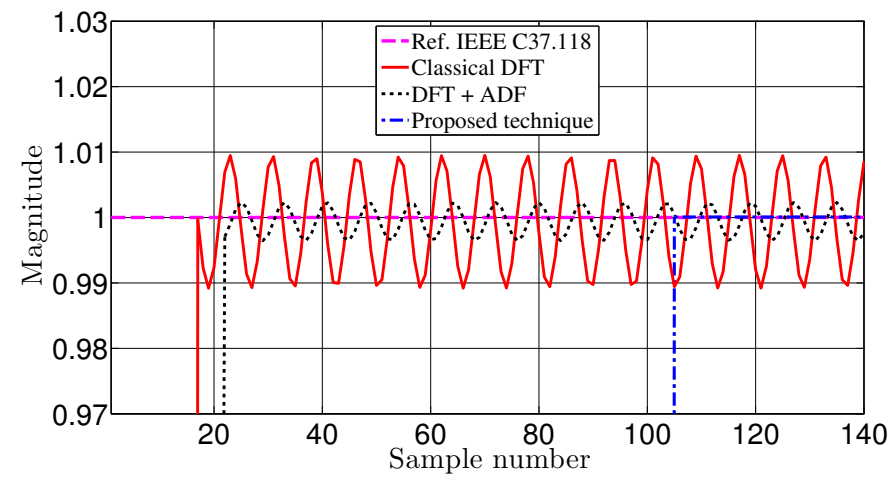

Fig. 5. Off-nominal synchrophasor magnitude estimate at $51 \mathrm{~Hz}$ according to IEEE Standard C37.118, classical full cycle DFT, DFT with six-sample ADF, and full span of the proposed technique

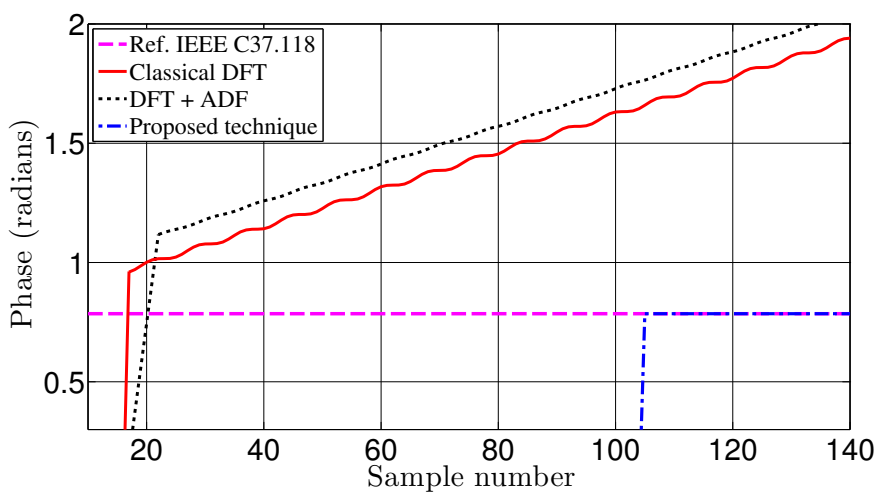

Fig. 6. Off-nominal synchrophasor angle estimate at $51 \mathrm{~Hz}$ according to IEEE Standard C37.118, classical full cycle DFT, DFT with six-sample ADF, and full span of the proposed technique

error. Therefore, it is required to perform magnitude, angle, and filtering compensations to allow that the classical DFT combined with ADF can comply with the IEEE Standard requirements. Once again, the synchrophasor angle estimate given by the proposed technique is free from oscillations and slope. Additional simulations with off-nominal signals have been performed whose results are compared with the reference synchrophasor to demonstrate the accuracy of the estimates, illustrated in Table II.

\section{CONCLUSION}

In this work, an enhanced adaptive data processing technique that combines in a synergistic manner frequency estimation based on WLS approach, FIR filtering process, linear interpolation using trigonometric identities, and half-cycle Radix-2 DIT FFT approach has been proposed for accurate computing of synchrophasor measurements under frequency variations. New techniques are envisaged to meet the need of reliable power system monitoring under transient conditions, therefore the synchrophasor estimates provided by the proposed technique, over the $\pm 3 \mathrm{~Hz}$ offset-nominal, demonstrate its capability in dealing efficiently with signals corrupted by spurious frequencies, being envisaged for potential applications involving M-Class PMU model.
TABLE II

OFF-NOMINAL SYNCHROPHASOR ESTIMATES

\begin{tabular}{cccc}
\hline \multirow{2}{*}{ Input signal } & \multicolumn{3}{c}{ Parameters } \\
\cline { 2 - 4 } & TVE (\%) & Magnitude (pu) & Angle (radians) \\
\hline $51.00 \mathrm{~Hz}$ & 0.0191 & 1.00007 & 0.78557 \\
$50.60 \mathrm{~Hz}$ & 0.0809 & 1.00004 & 0.78620 \\
$50.09 \mathrm{~Hz}$ & 0.0056 & 1.00004 & 0.78537 \\
$49.95 \mathrm{~Hz}$ & 0.0339 & 1.00009 & 0.78507 \\
$49.50 \mathrm{~Hz}$ & 0.0266 & 1.00020 & 0.78535 \\
$49.00 \mathrm{~Hz}$ & 0.0415 & 1.00041 & 0.78534 \\
\hline Ref. IEEE C37.118 & 0.0 & 1.0 & 0.78539 \\
\hline
\end{tabular}

\section{REFERENCES}

[1] A. G. Phadke and J. S. Thorp, Synchronized phasor measurements and their applications, New York: Springer, 2008.

[2] A. G. Phadke and R. M. de Moraes, "The wide world of wide-area measurement," IEEE Power and Energy Magazine, vol. 6, no. 5, pp. 52-65, Oct. 2008.

[3] Rodrigo J. Albuquerque and V. Leonardo Paucar, "Evaluation of the PMUs measurement channels availability for observability analysis," IEEE Trans. on Power Systems, vol. 28, no. 3, 2536-2544, Aug. 2013.

[4] W. Premerlani, B. Kasztenny, and M. Adamiak, "Development and implementation of a synchrophasor estimator capable of measurements under dynamic conditions," IEEE Trans. on Power Delivery, vol. 23, no. 1, 109-123, Jan. 2008.

[5] I. Kamwa, A. K. Pradhan, and G. Joos, "Adaptive phasor and frequencytracking schemes for wide-area protection and control," IEEE Trans. on Power Delivery, vol. 26, no. 2, pp. 744-753, Apr. 2011.

[6] I. Kamwa, S. R. Samantaray, and G. Joos, "Wide frequency range adaptive phasor and frequency PMU algorithms," IEEE Trans. on Smart Grid, vol. 5, no. 2, 569-579, Mar. 2014.

[7] P. Zhang, H. Xue, R. Yang and J. Zhang, "Shifting window average method for phasor measurement at offnominal frequencies," IEEE Trans. on Power Delivery, vol. 29, no. 3, pp. 1063-1073, Mai 2014.

[8] IEEE Standard for Synchrophasor Measurements for Power Systems, IEEE Std. C37.118.1-2011, Dec. 2011.

[9] G. D'Antona and A. Ferrero, Digital Signal Processing for Measurement Systems: Theory and Applications, New York: Springer, 2006.

[10] M. Karimi-Ghartemani, Boon-Teck Ooi, and A. Bakhshai, "Application of enhanced phase-locked loop system to the computation of synchrophasors," IEEE Trans. on Power Delivery, vol. 26, no. 1, pp. 22-32, Jan. 2011

[11] H. S. Malvar and D. H, Staelin, "Optimal FIR pre- and postfilters for decimation and interpolation of random signals," IEEE Trans. on Communications, vol. 36, no. 1, pp. 67-74, Jan. 1988.

[12] M. Akke and J. S. Thorp, "Sample value adjustment improves phasor estimation at off-Nominal frequencies," IEEE Trans. on Power Delivery, vol. 25, no. 4, pp. 2255-2263, Oct. 2010.

[13] T. S. Sidhu and M. S. Sachdev, "An iterative technique for fast and accurate measurement of power system frequency," IEEE Trans. on Power Delivery, vol. 13, no.1, pp. 109115, Jan. 1998.

[14] V. V. Terzija, M. B. Djuric, and B. D. Kovacevic, "Voltage phasor and local system frequency estimation using Newton type algorithm," IEEE Trans. on Power Delivery, vol. 9, no. 3, pp. 1368-1374, Jul. 1994.

[15] I. Kamwa and R. Grondin, "Fast adaptive schemes for tracking voltage phasor and local frequency in power transmission and distribution systems," Proc. of the IEEE Power Eng. Society - Transmission and Distribution Conference, pp. 930-936, Sept. 1991.

[16] Les Thede, Practical analog and digital filter design, Boston: Artec House, 2004.

[17] J. W. Cooley and J. W. Tukey, "An algorithm for the machine calculation of complex Fourier series," Mathematics of Computation, vol. 19, pp. 297-301, Apr. 1965

[18] L. Slosarcik (2013, Oct.). FFT-based algorithm for metering applications. Roznov Czech System Center. [Online]. Available: http://cache.freescale.com/files/32bit/doc/app_note/AN4255.pdf 